\section{Acknowledgments.}

Financial support. No financial support was provided relevant to this article.

Conflicts of interest. All authors report no conflicts of interest relevant to this article.

\section{References}

1. Elston J, Cartwright C, Ndumbi P, Wright J. The health impact of the 201415 Ebola outbreak. Public Health 2017;143:60-70.

2. Wang J, Zhou M, Liu F. Exploring the reasons for healthcare workers infected with novel coronavirus disease 2019 (COVID-19) in China. J Hosp Infect 2020; pii: S0195-6701(20)30101-8. doi: 10.1016/j.jhin.2020.03.002.

3. Ji Y, Ma Z, Peppelenbosch MP, Pan Q. Potential association between COVID-19 mortality and health-care resource availability. Lancet Global Health 2020;8(4):e480. doi: 10.1016/S2214-109X(20)30068-1.
4. Weinstein RS, Lopez AM, Joseph BA, et al. Telemedicine, telehealth, and mobile health applications that work: opportunities and barriers. Am J Med 2014;127:183-187.

5. Tuckson RV, Edmunds M, Hodgkins ML. Telehealth. N Engl J Med 2017;377:1585-1592.

6. Dorsey ER, Topol EJ. State of telehealth. N Engl J Med 2016;375:154-161.

7. Wang D, Hu B, Hu C, et al. Clinical characteristics of 138 hospitalized patients with 2019 novel coronavirus-infected pneumonia in Wuhan, China. JAMA 2020;323:1061-1069.

8. Wood CS, Thomas MR, Budd J, et al. Taking connected mobile-health diagnostics of infectious diseases to the field. Nature 2019;566:467-474.

9. Scott Kruse C, Karem P, Shifflett K, Vegi L, Ravi K, Brooks M. Evaluating barriers to adopting telemedicine worldwide: a systematic review. J Telemed Telecare 2018;24:4-12.

\title{
Antibiotic price rise and antibiotic stewardship programs-Stimulus or discouragement?
}

\author{
Felipe Francisco Tuon $\mathrm{PhD}^{1}$ (1), Joao Paulo Telles $\mathrm{MD}^{2}$, Juliano Gasparetto $\mathrm{PhD}^{3}$ and Tiago Zequinão Pharm ${ }^{1}$ \\ ${ }^{1}$ Laboratory of Emerging Infectious Diseases, Pontifícia Universidade Católica do Paraná, Curitiba PR, Brazil, ${ }^{2}$ Infectious Diseases Department, AC Camargo \\ Cancer Center, São Paulo, SP, Brazil and ${ }^{3}$ School of Medicine, Pontifícia Universidade Católica do Paraná, Curitiba PR, Brazil
}

To the Editor-An antimicrobial stewardship program (ASP) can be defined as the set of actions performed in hospitals for the rational use of antibiotics; reduction of adverse events, dosage errors, and appearance of multidrug-resistant bacteria; and shortening of length of hospital stay. The performance of the professional team implementing these programs, including clinical pharmacists, physicians and nurses, is associated with reduced in-hospital mortality rates. ${ }^{1}$ Managed antimicrobial administration programs shorten the length of hospital stay and reduce costs associated with the use of these medications.

Antibiotics account for a considerable cost in hospital bills, accounting for almost $20 \%$ of drug costs in Brazilian intensive care units. ${ }^{2}$ The usage policy is a controversial subject because it varies among hospitals. De-escalation of therapy and a switch from an intravenous to oral regimen, if it does not cause harm and demonstrates a safe strategy, can have an important outcome. ${ }^{3}$

ASPs are safe and cost-effective, an approach important particularly in developing countries. ${ }^{4}$ We demonstrated an estimated savings of US\$514,831 as a result of the implementation of the stewardship program in 2 institutions. These numbers encourage hospital administrators because there is always pressure to reduce costs. However, since the implementation of the ASPs in our hospitals, the price of medications has increased disproportionately. Figure 1 clearly shows the disproportionate increasing in the price of antimicrobials purchased by our group of hospitals in relation to various value indices.

The cost of some antibiotics increased $>500 \%$ over a period of 1 year (Fig. 1). This situation has led to a series of measures within

Author for correspondence: Felipe F. Tuon, E-mail: flptuon@gmail.com

Cite this article: Tuon FF, et al. (2020). Antibiotic price rise and antibiotic stewardship programs-Stimulus or discouragement?. Infection Control \& Hospital Epidemiology, 41: 994-995, https://doi.org/10.1017/ice.2020.125

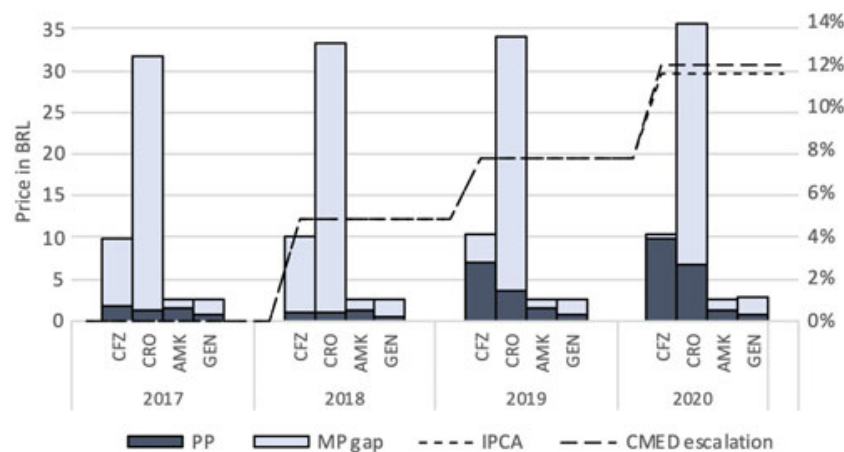

Fig. 1. Prices of 2 essential antibiotics, cefazolin (CFZ) and ceftriaxone (CRO), in the last 4 years, showing increasing costs, instead of aminoglycosides, amikacin (AMK), and gentamicin (GEN). The dark-gray columns indicate the price of each antibiotic and the light-gray columns indicate the maximum price allowed in Brazil. The dashed lines are the market index, IPCA for consumer prices, and CMED for drugs. Note. PP, purchase price; MP, maximum price; IPCA, Brazilian Broad Consumer Price Index; CMED, Brazilian Drugs Market Regulation Chamber.

our hospitals to contain costs to maintain the viability of our public hospital. We changed the surgical prophylaxis protocol from cefazolin to clindamycin. We implemented an active ASP for switching from an intravenous to an oral regimen as well as a de-hospitalization program (ie, outpatient antimicrobial therapy with oral and intravenous options). This cost crisis could be a stimulus to improve the ASP, but it will be discouraging if a cost reduction is not achieved.

In Brazil, the pricing of medicine is regulated by an agency called CMED (cf, Drug Market Regulation Chamber), which defines the maximum drugs prices that can be sold and the readjustment rates (Law no. 10.742, October 6, 2003). Annual escalation is calculated using an equation which IPCA (Broad 
Consumer Price Index), the official Brazilian inflation index, is the main variable (Decree no. 4,937, December 29, 2003). The accumulated CMED escalation amounted to $11.93 \%$ in the studied period, while the purchase prices of cefazolin and ceftriaxone (the antibiotics of choice for surgical prophylaxis and for treatment of pneumonia, urinary tract infections, meningitis, and intraabdominal infections) increased $617 \%$ and $292 \%$, respectively. On the other hand, amikacin and gentamicin, old drugs that are discouraged due to adverse reactions, suffered a $0.9 \%$ reduction and a $3 \%$ increase in the purchase price. Despite the disproportionate increase, the purchase prices did not exceed the maximum prices allowed by CMED. However, the annual trend suggests that the prices charged by laboratories and distributors will be very close to the maximum price allowed for widely marketed hospital antibiotics.

Recently, a small Missouri-based drug maker more than quadrupled the price of nitrofurantoin. ${ }^{5}$ In an interview, the chief executive said he had priced the product according to market dynamics and that it is a moral requirement to make money when you can. Furthermore, he said that this is a capitalist economy, and if you cannot make money, you cannot stay in business.

Clearly, antibiotic prices in Brazil are uncontrolled and antibiotics are in demand from suppliers. Antibiotics are considered the most important drugs in the treatment of serious infections. Many hospitals have avoided the most expensive antibiotics, but this may compromise the treatment of patients. Cost should be part of the
ASP, but it should not be the main engine of an amazing model of therapeutic rationalization.

Acknowledgments. None.

Financial support. No financial support was provided relevant to this article.

Conflicts of interest. All authors report no conflicts of interest relevant to this article.

\section{References}

1. Okumura LM, Silva MM, Veroneze I. Effects of a bundled antimicrobial stewardship program on mortality: a cohort study. Braz J Infect Dis 2015;19: 246-252.

2. Gasparetto J, Tuon FF, Dos Santos Oliveira D, et al. Intravenous-to-oral antibiotic switch therapy: a cross-sectional study in critical care units. BMC Infect Dis 2019;19:650.

3. Schuts EC, Hulscher M, Mouton JW, et al. Current evidence on hospital antimicrobial stewardship objectives: a systematic review and meta-analysis. Lancet Infect Dis 2016;16:847-856.

4. Boyles TH, Whitelaw A, Bamford C, et al. Antibiotic stewardship ward rounds and a dedicated prescription chart reduce antibiotic consumption and pharmacy costs without affecting inpatient mortality or re-admission rates. PLoS One 2013;8:e79747.

5. Crow D. Pharma chief defends $400 \%$ drug price rise as a 'moral requirement.' Financial Times website. https://www.ft.com/content/48b0ce2c-b544-11e8bbc3-ccd7de085ffe. Published September 11, 2018. Accessed April 20, 2020.

\title{
Achieving and maintaining low rates of hospital-onset Clostridioides difficile
}

\author{
Megan Rose Carr LaPorte MD $^{1}$ (D) and Lou Ann Bruno-Murtha DO ${ }^{1,2}$ \\ ${ }^{1}$ Cambridge Health Alliance, Cambridge, Massachusetts and ${ }^{2}$ Harvard Medical School, Boston, Massachusetts
}

To the Editor-The Cambridge Health Alliance (CHA) is a safety-net organization consisting of 277 beds spread between 2 community teaching hospitals and 3 emergency departments. We adopted polymerase chain reaction testing (PCR, Cepheid, Sunnyvale, CA) for Clostridioides difficile infection (CDI) in 2011 and, similar to other hospitals, we noted an increase in our hospital-onset ( $\mathrm{HO}) \mathrm{CDI}$ rate after adopting the more sensitive molecular assay. This increase occurred despite excellent hand hygiene practices, private rooms with contact precautions, daily bleach disinfection of high-touch surfaces, ultraviolet disinfection after terminal cleaning, and an antimicrobial stewardship program. A performance improvement project initiated in 2015 led our organization to successfully reduce the HO-CD standardized infection ratio (SIR) to $<1$ at 2 hospitals. ${ }^{1}$ We have been able to sustain a low HO-CD rate over the past 3.5 years despite changes in the National Healthcare Safety Network's (NHSN's) risk stratification methodology ${ }^{2}$ and without imposing additional testing restrictions on providers. In fact, our SIR has been maintained at $<1$ and has continued to decline.

Author for correspondence: Megan Rose Carr LaPorte, E-mail: mlaporte@cha. harvard.edu.

Cite this article: LaPorte MRC and Bruno-Murtha LA. (2020). Achieving and maintaining low rates of hospital-onset Clostridioides difficile. Infection Control \& Hospital Epidemiology, 41: 995-997, https://doi.org/10.1017/ice.2020.25

\section{Methods}

The initiation of an incentive program in 2015 led our institution to successfully implement a plan in February 2016 to drive the HO-CDI SIR to $<1$ via an automated nurse testing protocol (NTP). The goal of the NTP was to optimize identification of patients with community onset (CO) CDI to avoid inaccurate attribution of HO-CDI and inflation of the SIR due to a delay in stool specimen acquisition. In the NTP, which was embedded in Epic healthcare software (Verona, WI), documentation of diarrhea on the flowsheet during hospital days 1-3 led to a nurse best-practice alert that, when accepted, automatically led to stool collection and PCR testing of the specimen as well as initiation of contact precautions. We were careful to educate nurses and providers that a positive PCR test did not necessarily require antibiotic therapy because PCR detects $C$. difficile bacteria with the gene for toxin production but does not detect the toxin itself. Patients with recent risk factors for CDI or clinical characteristics of disease (eg, fever, severe diarrhea, or leukocytosis) were started on treatment, whereas those who had other reasons for diarrhea (eg, medications, diet, or laxatives) were advised to continue with watchful waiting.

In July 2016, a combined glutamate dehydrogenase (GDH) antigen and toxin assay for toxins A and B (Abbott Diagnostics, 